\title{
A flexible and accurate method to estimate the mode and stability of spontaneous coordinated behaviors: The index-of-stability (IS) analysis
}

\author{
Gregory Zelic $^{1}$ - Deborah Varoqui ${ }^{2} \cdot$ Jeesun Kim $^{1} \cdot$ Chris Davis $^{1}$
}

Published online: 24 February 2017

(C) Psychonomic Society, Inc. 2017

\begin{abstract}
Patterns of coordination result from the interaction between (at least) two oscillatory components. This interaction is typically understood by means of two variables: the mode that expresses the shape of the interaction, and the stability that is the robustness of the interaction in this mode. A potent method of investigating coordinated behaviors is to examine the extent to which patterns of coordination arise spontaneously. However, a prominent issue faced by researchers is that, to date, no standard methods exist to fairly assess the stability of spontaneous coordination. In the present study, we introduce a new method called the index-of-stability (IS) analysis. We developed this method from the phase-coupling (PC) analysis that has been traditionally used for examining locomotion-respiration coordinated systems. We compared the extents to which both methods estimate the stability of simulated coordinated behaviors. Computer-generated time series were used to simulate the coordination of two rhythmic components according to a selected mode $m: n$ and a selected degree of stability. The IS analysis was superior to the PC analysis in estimating the stability of spontaneous coordinated behaviors, in three ways: First, the estimation of stability itself was found to be more accurate and more reliable with the IS analysis. Second, the IS analysis is not constrained by the limitations of the PC analysis. Third and last, the IS analysis
\end{abstract}

Electronic supplementary material The online version of this article (doi:10.3758/s13428-017-0861-2) contains supplementary material, which is available to authorized users.

Gregory Zelic

gregory.zelic@gmail.com

1 The MARCS Institute, Western Sydney University, Sydney, NSW, Australia

2 Euromov, University of Montpellier, Montpellier, France offers more flexibility, and so can be adapted according to the user's needs.

Keywords Spontaneous entrainment · Frequency-locking · Phase-coupling $\cdot$ Stability $\cdot$ Method

Coordination is ubiquitous in our daily-life actions (Kelso, 1995; Nourrit-Lucas, Zelic, Deschamps, Hilpron, \& Delignières, 2013; Turvey, 1990). Research in the human movement sciences has shown entrainment between breathing and locomotion (Bramble \& Carrier, 1983; McDermott, Van Emmerik, \& Hamill, 2003), between the movements of our limbs (Kelso, 1995; Zelic, Mottet, \& Lagarde, 2012, 2016), between speech and gesture (Shockley, Baker, Richardson, \& Fowler, 2007; Zelic, Kim, \& Davis, 2015), between the postures of people (Lagarde, 2013; Varlet, Marin, Lagarde, \& Bardy, 2011), and between a person's movements and external cues (Repp \& Su, 2013; Zelic, Varlet, Kim, \& Davis, 2016).

One elegant approach for understanding the formation and evolution of such coordinated behaviors was developed in the early eighties on the basis of dynamic-systems theory and the principle of self-organization. According to this view, the emergence and change of coordination patterns in biological systems result from the functional interactions of the system's components to ensure the system's robustness (Haken, 2013; Haken, Kelso, \& Bunz, 1985; Kelso, 1995; Turvey, 1990). These interactions are constrained both by the intrinsic properties of the system's components and by its tolerance to perturbations, either those internal to the system-for example, biological noise - or those due to environmental influences. This approach provides a potent method to investigate coordination dynamics by measuring the extent to which patterns of coordination arise spontaneously. Spontaneous coordinated patterns are indeed characterized by a lack of intentional 
forcing, which minimizes the strength of the interaction established within the coordinative system. That is, spontaneous patterns of coordination are more likely to respond to minimal changes and constraints - for example, those manipulated in experimental protocols.

Despite this advantage of spontaneous coordination for experimentation, the bulk of research has investigated intentional patterns of coordination (e.g., in human movement research); we argue that a reason for this may be the lack of a proper method to estimate with accuracy the mode and stability of spontaneous coordinated behaviors. In the following sections, we will briefly consider what the mode and stability are and why these are more challenging to estimate for spontaneous than for intentional coordination patterns.

The mode, or frequency-locking, can be thought of as the shape of the coordination. It consists in the rational ratio $m: n$ in which the fundamental frequencies $\omega$ and $\Omega$ of two rhythmic components spontaneously lock when interacting within a coordinative system, such as $n \omega=m \Omega$ (Guevara \& Glass, 1982; Pikovsky, Rosenblum, \& Kurths, 2003). It reflects the mutual entrainment of the coupled components, the so-called resonance response of the coordinative system that occurs whenever the frequency of a harmonic of one component $(n \omega)$ approaches some harmonic of the other $(m \Omega)$, where $n$ and $m$ are positive integers.

Stability refers to the strength of the interaction - that is, how robust the coordinative system is within a mode. It reflects the strength of the intrinsic coupling within the coordinative system: the stronger the coupling, the more stable the coordination. A typical measure of coordination stability is given by the fluctuations over time of the relative phase - that is, the difference between the phase of one component and that of the other. When coordination is established, it is established in a particular mode of coordination $m: n$ in which the phases of the coupled components are locked together. In this case, the relative phase is bounded such that:

$m \theta_{B}-n \theta_{A} \mid<$ constant

where $\theta_{A}$ and $\theta_{B}$ are the phases of the components $\mathrm{A}$ and $\mathrm{B}$ that are coupled within the $m: n$ mode of coordination (Pikovsky et al., 2003; Tass et al., 1998). The degree of fluctuation of the relative phase then indicates the extent to which the coordinated system is stable within this mode of coordination: the less variable the relative phase, the more stable the coordination. Note that in the absence of coupling, the phases of the components evolve independently from each otherthat is, diverge, and thus the relative phase grows indefinitely.

From the definitions above and Eq. 1, it is clear that stability is estimated with respect to the mode of the coordination. That is, a requirement for estimating stability is to determine a priori in which $m: n$ mode the coordination has been established. Within an intentional coordination paradigm in which participants are instructed to produce a particular pattern of coordination, the usual procedure is to estimate stability with respect to the mode instructed to be performed in the experimental task. The problem is that for spontaneous coordination patterns, by definition, no mode of coordination is instructed to be performed. That is, no $m$ and $n$ integers are identified prior to the calculations of relative phase and the estimation of its fluctuations. To date, no standard methods exist for estimating stability in such scenarios. Previous research has typically estimated the stability of spontaneous coordinated behaviors by extracting the mode of coordination, or the $m$ and $n$ integers, by trial and error (Kelso \& Jeka, 1992; Pikovsky et al., 2003). In general terms, the following procedure would be applied: (1) A relative phase time series would be computed regardless of the mode of coordination - that is, a standard relative phase time series; (2) the fluctuations of this standard relative phase would be estimated with respect to different modes $m: n$ arbitrarily selected according to the expectations of the researcher as to what modes might occur in the dataset; and (3) the mode for which the fluctuations of this standard relative phase were minimal would be identified as the mode of coordination, and stability would be estimated from the fluctuations of the relative phase with respect to this mode.

On the basis of the procedure above, a method known as phase-coupling (PC) analysis was developed. Importantly, this method proposed a more principled way to select the $m: n$ modes, which had to be chosen arbitrarily in the original procedure (see PC Step 2 in S1 Appendix). Under the PC analysis, potential $m: n$ modes are selected according to the successive levels of the Farey tree (Cvitanovic, Shraiman, \& Söderberg, 1985; Hardy \& Wright, 1979; see Fig. 1). The Farey tree ranks all potential modes of coordination from lower-order modes - that is, modes with small integers $m: n$, such as 1:1 (Level 1 of the Farey tree) - to higher-order modes - that is, modes with larger integers $m: n$, such as 2:5 (Level 4 of the Farey tree; see Fig. 1). It provides a general structure for understanding the dynamics of the mode-locking

\section{Level}

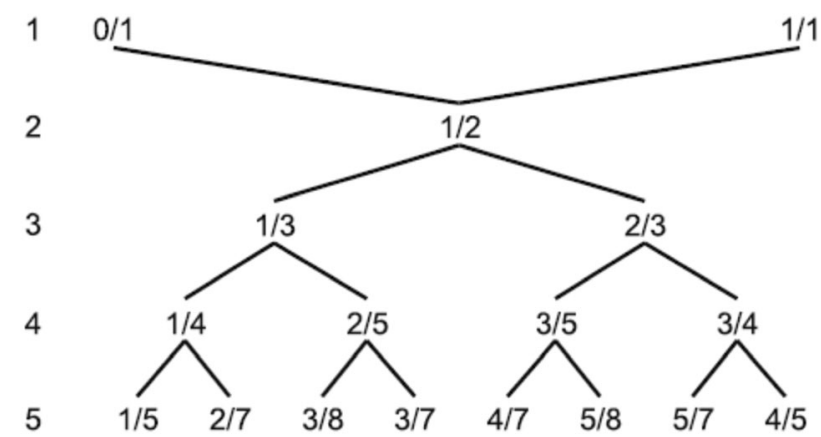

Fig. 1 Representation of the first five levels of the Farey tree. From "Sound Stabilizes Locomotor-Respiratory Coupling and Reduces Energy Cost," by C. P. Hoffmann, G. Torregrosa, and B. G. Bardy, 2012, PLoS ONE, 7, e45206. Copyright 2012 by Hoffmann et al. Reprinted with permission 
behavior of a coordinative system, as predicted by a nonlineardynamics model known as the circle map model (Jensen, Bak, \& Bohr, 1984; Kelso, 1991, 1995; Pikovsky et al., 2003; Treffner \& Turvey, 1993; see S4 Appendix). Briefly, this mathematical model anticipates the extent to which a mode of coordination can be performed, given the initial conditions of two coupled components and the strength of their interaction. In particular, the model favors lower-order modes with larger regions of attraction, and therefore with greater chances to be spontaneously performed, in comparison to higher-order modes. Research on human coordinated behavior has provided support for the circle map model, since it is classically observed that lower-order ratios are more frequently produced and are more stable (Kelso \& de Guzman, 1988; Peper, Beek, \& van Wieringen, 1995; Treffner \& Turvey, 1993).

The PC analysis has been used extensively over the last two decades, mostly to examine coordination between the locomotory and respiratory systems (Hamill, McDermott, \& Haddad, 2000; Hoffmann, Torregrosa, \& Bardy, 2012; Kiefer, Riley, Shockley, Villard, \& Van Orden, 2009; McDermott et al., 2003; O'Halloran, Hamill, McDermott, Remelius, \& Van Emmerik, 2012; Peper et al., 1995; Van Emmerik, Rosenstein, McDermott, \& Hamill, 2004; Villard, Casties, \& Mottet, 2005; see S1 Appendix). In this respect, the PC analysis can be seen as the default standard in the area.

However, to date, no study has been conducted to empirically validate the use of the PC analysis for estimating the mode and stability of spontaneous coordination. That is, the extent to which the stability score that is given by the PC analysis relates to the stability of the actual coordination is still poorly understood. In addition, a close examination of the PC analysis indicated that its calculations are based on assumptions that are unlikely to apply in many instances of spontaneous coordination. We determined three limitations to the generalization of the PC analysis to spontaneous coordination. First, the PC analysis is asymmetric: The calculations require identifying the coupled components either as target or as reference. This necessary selection is arbitrary in the case of spontaneous coordination, and this is problematic because the stability score given via the PC analysis depends on this selection. Second, the PC analysis is not sensitive to changes in the mode of coordination. That is, stability is estimated with respect to one mode of coordination only, the one most dominant within the period of observation. This is another issue for nonintentional coordination tasks in which more than one mode of coordination is likely to be performed. Third, the PC analysis is not sensitive to small phase drift- that is, a minimal and constant change in the relative phase values. This last limitation is not specific to spontaneous coordination, and is directly related to the return map method used to estimate the dispersion of relative phase. In the S2 Appendix, we detail the extent to which each of these limitations can affect the stability score estimated with the PC analysis.
In the present study, we aimed to establish and to empirically validate a method for accurately estimating the mode and the stability of spontaneous coordinated behaviors. To do so, we developed the index-of-stability (IS) analysis as an alternative approach that overcomes the above limitations of the $\mathrm{PC}$ analysis (S3 Appendix). That is, the IS analysis proposes an estimation of the stability of spontaneous coordinated behaviors that is not constrained by any of the highlighted limitations of the PC analysis.

Both methods fundamentally differ in the ways that the mode of coordination is used in the calculations of stability. The PC analysis computes a standard relative phase time series that is independent of the mode of coordination, then estimates stability by assessing the fluctuations of this standard relative phase with respect to a mode of coordination. A range of modes are tried out, and the one leading to the highest stability score is selected as the mode of the coordination (S1 Appendix). The IS analysis, in contrast, computes a moderelated relative phase time series that takes into account the modes of coordination produced locally. That is, the mode produced at the instant $t$ is used to calculate the relative phase value at the instant $t$. Here, there is no need to select a mode of coordination to assess the fluctuations of the relative phase; stability is derived directly from the circular dispersion of the relative phase values (see S3 Appendix).

Given this difference in calculations between the two methods, the question arises of whether the IS analysis would perform as well as the PC analysis for cases that do not involve any of the three limitations identified for the PC analysis (see above). To examine this question, we used a computergenerated time series to simulate the coordination of two rhythmic components $\mathrm{A}$ and $\mathrm{B}$ and compared the extents to which the two methods can estimate the stability of chosen $m: n$ modes performed at chosen degrees of stability. This investigation is important for two reasons: (1) It is essential to determine whether the modifications that enable the IS analysis to be more general than the PC analysis come at a cost, and (2) any future research will require the empirical validation of the proposed method to determine the extent to which it is able to accurately estimate coordination stability.

\section{Materials and method}

\section{Data simulation}

The data simulation procedure is outlined in Fig. 2. The figure illustrates the procedure used for the creation of the simulated time series data. The vertical bars represent the simulated time onsets for the coupled components A and B. At first, the time onsets of the component $\mathrm{A}$ and the component $\mathrm{B}$ were determined to simulate in-phase coordination on a given $m: n$ mode. We maintained the time onsets of the component $A$ across the 


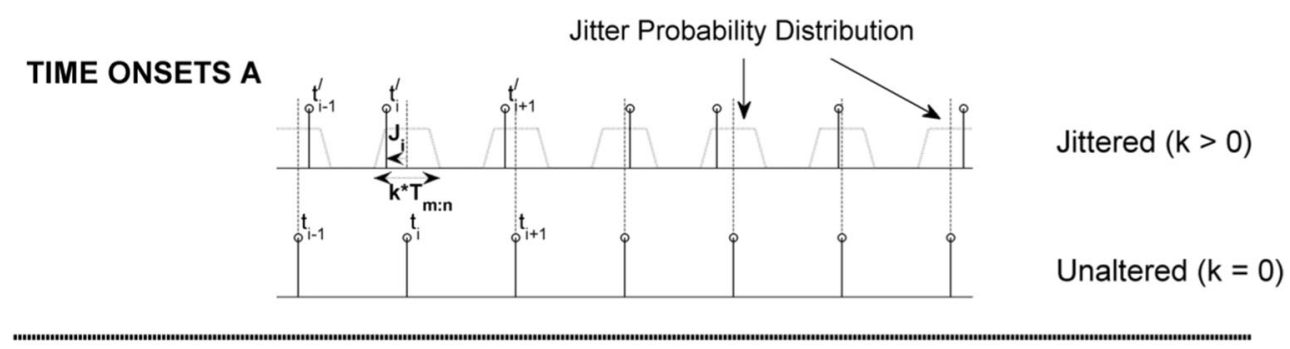

TIME ONSETS B

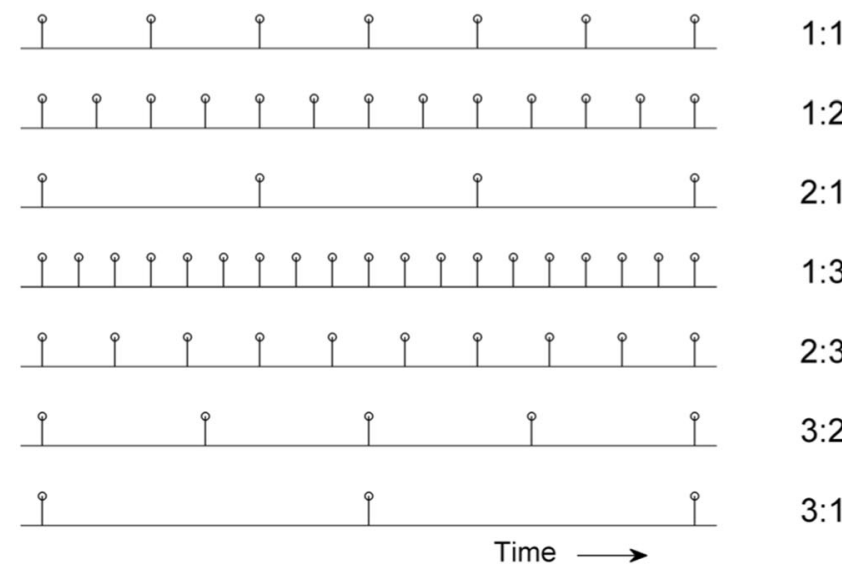

Fig. 2 Data simulation. The time onsets of the oscillators A and B were first determined to simulate perfect in-phase coordination on chosen $m: n$ modes $(1: 1,1: 2,2: 1,1: 3,2: 3,3: 2$, and $3: 1)$. The stability of the coordination was then manipulated by adding a temporal jitter $J_{i}$ to the

different modes tested; only the time onsets of the component $B$ varied as a function of the mode of the coordination (see the next section). Second, the time onsets of the component A were manipulated to simulate a certain degree of stability of the coordination (see the following sections on periodicity and the jitter function).

In-phase coordination on an m:n mode The time series $t_{i}$ and $t_{j}$ were determined by considering the perfect in-phase coordination of the oscillators $\mathrm{A}$ and $\mathrm{B}$ according to one targeted $m: n$ mode. Seven modes were targeted, all taken from Level 1 to Level 3 of the Farey tree: 1:1, 1:2, 2:1, 1:3, 2:3, 3:2, and 3:1. The oscillator A was considered to oscillate at the same rate, $F r$, for $M=1,000$ cycles, regardless of what the targeted $m: n$ mode was. That is, only the time onsets $t_{j}$ of the oscillator B varied as a function of the targeted mode (see Fig. 2). For instance, B would complete $N=1,000$ cycles at the same rate $F r$ as A for a 1:1 mode, or $N=2,000$ cycles at twice the Fr rate for a 1:2 mode. At this stage, we can write:

$\theta_{\mathrm{A}}\left(t_{i}\right)=\theta_{\mathrm{B}}\left(t_{j}\right)=0+i, j[2 \pi]$,

with $i: 0 \rightarrow M=1,000$ and $j: 0 \rightarrow N$, where $\theta_{A}\left(t_{i}\right)$ and $\theta_{B}\left(t_{j}\right)$ are the phases of the coupled oscillators A and B at the times $t_{i}$ and $t_{j}$, respectively, and $M$ and $N$ are the total numbers of complete cycles for the oscillators A and B over a trial. time onsets $t_{i}$ of the oscillator A: the larger $J_{i}$ the less stable the coordination. $J_{i}$ is defined as a random value taken from a uniform distribution whose base is function of the chosen $m: n$ mode and of coordination stability (see the text for details)

Constraints in manipulating coordination stability: Periodicity of the relative phase The variability of the time onsets of component $\mathrm{A}, t_{i}$, was then manipulated to simulate the degree of stability of the coordination. The jitter $J_{i}$ was defined such that the resultant time onset $t_{i}$ was defined as

$t_{i}^{\prime}=t_{i}+J_{i}$

Whereas the time series $\left\{t_{i} ; t_{j}\right\}$ simulated a perfect in-phase coordination of $\mathrm{A}$ and $\mathrm{B}$ within a certain mode $m: n$, characterized by a relative phase distributed Dirac-like around 0 , the time series $\left\{t_{i}^{\prime} ; t_{j}\right\}$ simulated the less stable $m: n$ coordination characterized by a relative phase uniformly distributed within a $\left[-k^{*} \pi ; k^{*} \pi\right]$ range, $k \in[0 ; 1]$.

One particular constraint in manipulating coordination stability is related to the jitter $J_{i}$, because the same jitter $J_{i}$ will differently shift the relative phase value $R P\left(\dot{t}_{i}\right)$ depending on the mode $m: n$ of the coordination. Consider, for instance, the perfect in-phase coordination within the modes $1: 1$ and $1: 2$; that is, component A completes one cycle while component B completes, respectively, one and two cycles. Regardless of the mode, the time onsets $t_{j}$ of component B occur systematically at the same time as some time onset $t_{i}$ of component $\mathrm{A}$. That is, the phase of the oscillator B at the time onsets $t_{i}$ is $\theta_{B}\left(t_{i}\right)=\theta_{B}\left(t_{j}\right)=0[2 \pi]$, and as a consequence, $\operatorname{RP}\left(t_{i}\right)=0[2 \pi]$. Now applying the jitter $J_{i}=\operatorname{period}(A) / 2$, the resultant time onset $\dot{t}_{i}$ reads $\dot{t}_{i}=t_{i}+\operatorname{period}(A) / 2$. For a $1: 1$ mode, the resultant 
time onset $t_{i}$ is perfectly interposed between $t_{j}$ and $t_{j+1}$ - that is, the phase of the oscillator B at $t_{i}$ is $\theta_{B}\left(\dot{t}_{i}\right)=\pi[2 \pi]$, and $\operatorname{RP}\left(\dot{t}_{i}\right)=\theta_{B}\left(\dot{t}_{i}^{\prime}\right)-\theta_{A}\left(\dot{t}_{i}\right)=\pi[2 \pi]$. In comparison, for a 1:2 mode, the resultant time onset $t_{i}$ becomes perfectly aligned with $t_{j+1}$. In this case, $\theta_{B}\left(\dot{t}_{i}^{\prime}\right)=\theta_{B}\left(t_{j+1}\right)=2 \pi[2 \pi]$, and as a consequence, $\operatorname{RP}\left(\dot{t}_{i}^{\prime}\right)=\theta_{B}\left(\dot{t}_{i}^{\prime}\right)-2 * \theta_{A}\left(t_{i}^{\prime}\right)=2 \pi[2 \pi]$. In other words, at the time onset $t_{i}$, the same jitter $J_{i}$ leads to phase shifts of $\pi$ and $2 \pi$ in the relative phase distributions, respectively, for a $1: 1$ and a 1:2 mode. These differences in phase shift for the same temporal deviation result from the difference in periodicity of the relative phase as a function of the mode of coordination $m: n$. For $1: 1$, the relative phase completes one revolution for $T_{1: 1}=\operatorname{period}(A)=\operatorname{period}(B)$, whereas for $1: 2, T_{1: 2}=\frac{\operatorname{period}(A)}{2}$ $=\operatorname{period}(B)$. A general expression to capture the periodicity of the relative phase when $\mathrm{A}$ and $\mathrm{B}$ are locked into an $m: n$ mode is

$T_{m: n}=\frac{\operatorname{period}(A)}{n}=\frac{\operatorname{period}(B)}{m}$.

The jitter function $T_{m: n}$ expresses the maximal time window in which the time onsets of the component A can be jittered. That is, for each targeted $m: n$ mode, we can vary the time onsets $t_{i}$ of the component A within the $\left[t_{i}-k * \frac{T_{m: n}}{2} ; t_{i}+k * \frac{T_{m: n}}{2}\right]$ range, such that the resultant relative phase at the time onsets $t_{i}$ is uniformly distributed within a $\left[-k^{*} \pi ; k^{*} \pi\right]$ range, $k \in[0 ; 1]$. In other words, we can define the jitter $J_{i}$ in Eq. 3 as a random value from the uniform distribution bounded within the $\left[-k * \frac{T_{m: n}}{2} ; k * \frac{T_{m: n}}{2}\right]$ range. $J_{i}$ was therefore determined such that the resultant time onset $\dot{t}_{i}$ reads $\dot{t}_{i}^{\prime}=t_{i}+J_{i}(m: n, k)$, with

$J_{i}(m: n, k)=R_{i} *\left(k * \frac{T_{m: n}}{2}\right)$,

$i: 1 \rightarrow M . R_{i}$ corresponds to the $i$ th value of a uniform distribution of $M$ elements bounded within the $[-1 ; 1]$ range. Note that $R_{i}$ is consistent for whatever mode $m: n$ and $k$ considered. $T_{m: n}$ is the period of the relative phase for the targeted $m: n$ mode, and $\frac{T_{m: n}}{2}$ represents the maximal amplitude of the jitter. $k$ varies from 0 to 1 in order to vary the maximal jitter amplitude from 0 to $\frac{T_{m: n}}{2}$. As a result, the resultant relative phase time series at the jittered time onsets $t_{i}$, regardless of the targeted $m: n$ mode, is distributed within the $\left[-k^{*} \pi ; k^{*} \pi\right]$ range. For $k=0, t_{i}=t_{i}$, and the relative phase is distributed Dirac-like such that the phase coupling is maximal and the coordination perfectly stable. In contrast, for $k=1, t_{i}$ is distributed within the maximum range $\left[t_{i}-\frac{T_{m: n}}{2} ; t_{i}+\frac{T_{m: n}}{2}\right]$. As a consequence, the resultant relative phase is uniformly distributed on a $2 \pi$ interval, reflecting a no-phase-locking scenario- that is, no coordination.

\section{Method}

Procedure One simulation as described above creates $X=357$ simulated time series $\left\{\dot{t}_{i}(k, m: n) ; t_{j}(m: n)\right\}$ - that is, one for each of the seven modes targeted $(1: 1,1: 2,2: 1,1: 3,2: 3,3: 2$, $3: 1)$ crossed with each of the 51 levels of $k$, such that $k: 0 \rightarrow 1$ by 0.02 steps. We performed 50 simulations, each one using a different $\mathrm{R}$ vector. Each of the time series $\left\{t_{i}^{\prime}(k, m: n) ; t_{j}(m: n)\right\}$ was then analyzed by using the PC analysis and the IS analysis. Both methods require selecting a level in the Farey tree at which the analysis is stopped. We selected Level 10 of the Farey treethat is, the analysis was performed for both methods from $F_{1}$ to $F_{10}$. Since the modes of coordination simulated in the present experiment where taken from Level 1 to Level 3 of the Farey tree, we could have selected any level from Level 3 (Fig. 1). Level 10 was selected arbitrarily, but this selection had no impact on the present comparative study since it was selected for both analyses. A detailed description of the Farey tree is given in S1 Appendix. We examined whether and to what extent each technique could retrieve the correct mode $m: n$ and the correct degree of stability chosen for simulating the time series.

Comparison to the predicted PC and the predicted IS As we mentioned in the description above, the degree of stability of the coordination was simulated by using the parameter $k$, such that whatever the mode of the coordination $m: n$ chosen in the simulation, the resultant relative phase was uniformly distributed within the $\left[-k^{*} \pi ; k^{*} \pi\right]$ range, $k \in[0 ; 1]$. That is, the estimation of stability did not depend on the chosen $m: n$ mode, but only on the parameter $k$. It was therefore possible to predict the correct stability score that should be estimated by the PC analysis and by the IS analysis as a function of $k$ by considering $\theta$ uniformly distributed on a $\left[-k^{*} \pi ; k^{*} \pi\right]$ range with $k: 0 \rightarrow 1$, increasing by steps of 0.02 . The prediction made from the PC analysis, $P C_{\text {pred }}(k)$, was given by the estimation of the fluctuations of $\theta$ distributed on $\left[-k^{*} \pi ; k^{*} \pi\right]$ with the return map technique (PC Step 3 in S1 Appendix). This prediction should match the stability score $P C(k)$ estimated when processing the simulated time series $\left\{\dot{t}_{i}(k, m: n) ; t_{j}(m: n)\right\}$ with the PC analysis. Similarly, the prediction made from the IS analysis, $I S_{\text {pred }}(k)$, was given by the estimation of the fluctuations of $\theta$ distributed on [-k* $\left.\pi ; k^{*} \pi\right]$ with the mean resultant length technique (IS Step 3 in S3 Appendix). This prediction should match the stability score $I S(k)$ estimated when processing the simulated time series $\left\{t_{i}(k, m: n) ; t_{j}(m: n)\right\}$ with the IS analysis. That is, whatever the mode $m: n$ chosen for the simulation, we should have $P C(k)=P C_{\text {pred }}(k)$ and $I S(k)=I S_{\text {pred }}(k)$.

To determine $P C_{\text {pred }}(k)$ and $I S_{\text {pred }}(k)$, we computed 10,000 simulations of $\theta$ uniformly distributed on $[-k * \pi ; k * \pi]$ with $N_{\theta}=1,000$, where $N_{\theta}$ is the number of values in $\theta . P C_{\text {pred }}(k)$ and $I S_{\text {pred }}(k)$ were computed as the average estimations of the dispersion of $\theta(k)$ by using the return map technique (PC Step 3 in S1 Appendix) and the mean resultant length technique (IS 
Step 3 in S3 Appendix), respectively. The results are shown in Fig. 3. Note in Fig. 3 the difference in the stability scores estimated by the two methods. Both methods aim to estimate the fluctuations of the exact same distribution $\theta$, yet they do so in different ways.

\section{Results}

\section{Estimation of coordination stability}

As predicted, the stability scores estimated as a function of $k$ when processing the simulated time series $\left\{t_{\mathrm{i}}(k, m: n) ; t_{\mathrm{j}}(m\right.$ : $n)\}$ differed depending on the method used. The stability scores estimated via the PC analysis show similar shapes across the $m: n$ modes tested (Fig. 4, left panel). As expected, $P C=1$ when $k=0$ - that is, when no jitter was applied to $t_{i}$ and the $m: n$ coordination was simulated with perfect phase coupling. Then a linear drop occurs within the $\left[k ; k^{\prime}\right]$ interval, where the estimated $P C$ drops below the predicted $P C_{\text {pred. }}$. Note that the sizes of the drop differ across the $m: n$ modes tested. Finally, the estimated $P C$ function is characterized by an asymptotic regime from $k=k^{\prime}$ to $k=1$.

The stability scores estimated via the IS analysis also show similar shapes across the $m: n$ modes tested, with an apparent better match to the prediction $I S_{\text {pred }}$ (Fig. 4, right panel). Accordingly, we found that $I S(k)$ was a better fit with $I S_{\text {pred }}(k)$ than was $P C(k)$ with $P C_{\text {pred }}(k)$ (Fig. 5). First, for any value of $k$, the absolute error between the IS stability scores estimated by IS

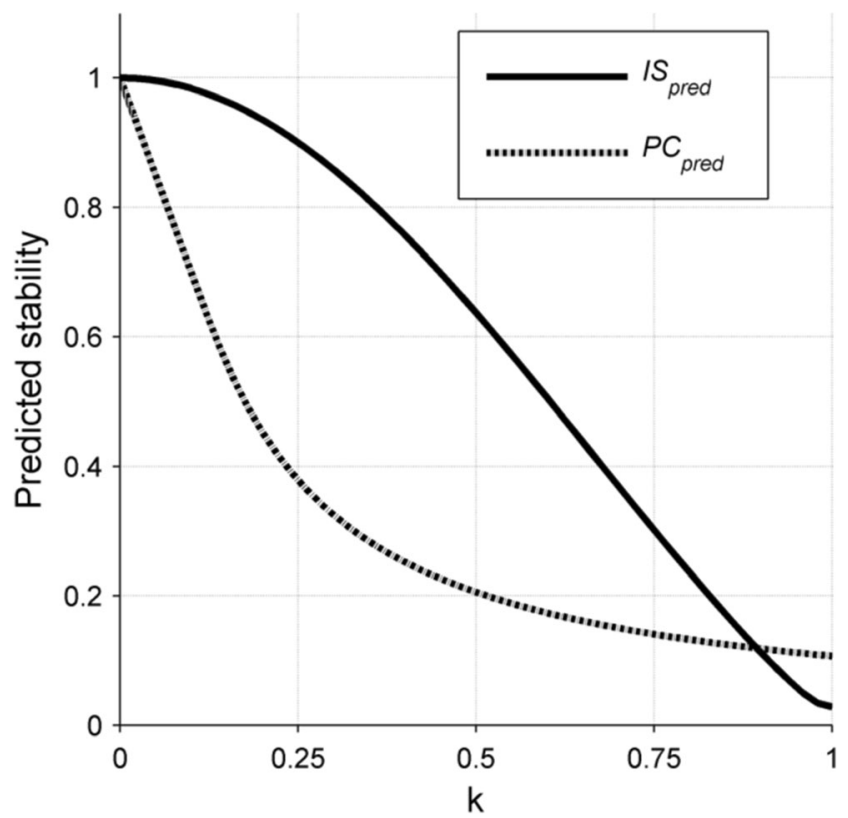

Fig. 3 Predicted stability scores with the IS analysis $\left(\mathrm{IS}_{\text {pred }}\right)$ and the PC analysis $\left(\mathrm{PC}_{\text {pred }}\right)$ as a function of the chosen degree of stability $(k)$. The figure illustrates the predicted stability scores estimated from the fluctuations of $\theta$ uniformly distributed on the interval $\left[-k^{*} \pi ; k^{*} \pi\right]$ with the IS analysis (solid line) and with the PC analysis (dotted line) analysis and those predicted ( $\left.I S_{\text {pred }}\right)$ was systematically smaller than the absolute error between the stability scores from the PC analysis - that is, between estimated $P C$ and predicted $P C_{\text {pred }}$ (Fig. 5, left panel). The absolute error between the estimated and predicted stability scores was on average systematically smaller than 0.1 for the IS analysis. In contrast, for the PC analysis, the absolute error between the estimated and predicted stability scores was on average greater than 0.1 for $k>0$. Second, the correlation between the functions of the stability scores estimated and predicted across the seven $m: n$ modes is on average higher with the IS analysis (mean $[M]=.99,95 \%$ confidence interval $[\mathrm{CI}]=.004)$ than with the $\mathrm{PC}$ analysis $(M=.92, \mathrm{CI}=$ .054 ; see Fig. 5 , right panel).

In addition, the results showed that the stability scores estimated as a function of $k$ across the different $m: n$ modes tested were more consistent with the IS analysis than with the PC analysis. Accordingly, the standard deviation of the $P C(k)$ values estimated at each $k$ for the seven $m: n$ modes tested via the $\mathrm{PC}$ analysis was found to be greater than the standard deviation of the $I S(k)$ values estimated at each $k$ for the seven $m: n$ modes tested via the IS analysis (Fig. 6).

\section{Recognition of the m:n mode chosen for the data simulation}

Table 1 presents, in percentages, the ratios of correct identification of the m:n mode used for the time series over the 50 simulations performed for each $k$. The PC analysis can systematically identify the mode used for the simulated data as the dominant mode of the coordination, even for extreme values as $k$ tends to 1 . The IS analysis can also identify the $m: n$ mode chosen for the simulated data as the dominant one from $k=0$ to $k=0.88$, but it is not $100 \%$ correct for larger value of $k$ in the case of the highest-order modes of the series - that is, $3: 1,3: 2,1: 3$, and $1: 2$. Note that for each $k$, the percentage is still greater than $50 \%$, which means that the mode chosen for the data simulation was always identified for more than half of the 50 simulations.

\section{Discussion}

In the present study, we compared the extents to which the PC analysis and the IS analysis estimate the mode and stability of spontaneous coordinated behaviors. We tested the accuracythat is, how precise the estimation is - and the reliability - that is, how consistent the estimation is as a function of the mode $m: n$ - using computer-generated time series that simulated the coordination of two rhythmic components according to a selected mode $m: n$ and a selected degree of stability. Note that the simulation was set up so that it did not involve the limitations identified for the PC analysis (i.e., changes in coordination mode were not simulated, nor was phase drift; 


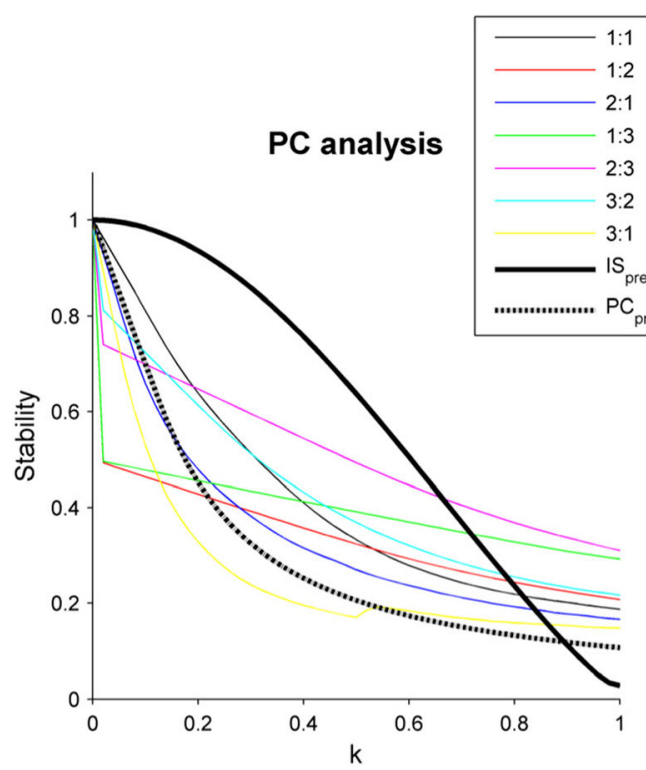

Fig. 4 Stability scores as a function of $k$ for each $m: n$ mode chosen in the simulated time series $\left\{\dot{t}_{i}(k, m: n) ; t_{j}(m: n)\right\}$. The stability score was estimated by either the PC analysis (left panel) or the IS analysis (right

see Limitations 2 and 3 in the S2 Appendix and S3 Appendix) as including such would have clearly disadvantaged the PC analysis. The results showed that even in these conditions, the IS analysis provides a more accurate and a more reliable estimate of the coordination stability. First, the IS analysis resulted in a more precise estimate of stability than the PC analysisthat is, the estimated stability was a better fit to the model. Second, the IS analysis gave more consistent estimates of stability across the different $m: n$ modes tested than did the PC analysis. This is important, since a method dedicated to estimation of the stability of a coordination pattern should not be affected by the mode $m: n$ in which the coordination is locked.

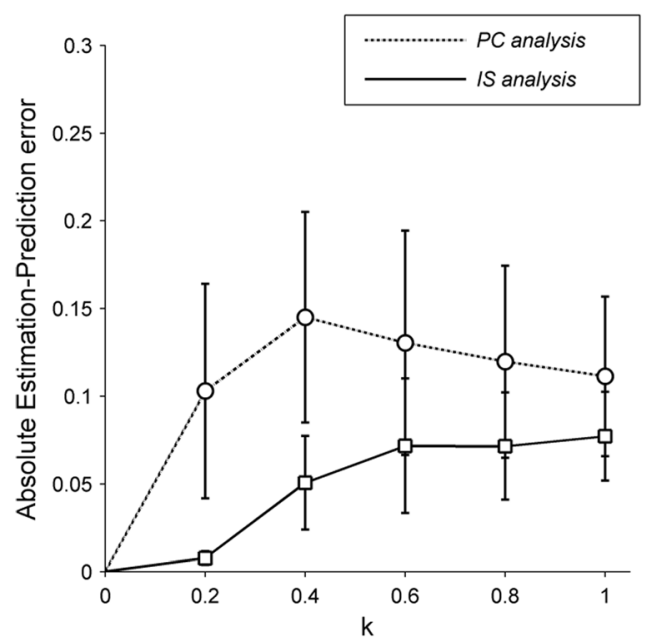

Fig. 5 Differences between the stability scores predicted and estimated for the PC analysis and for the IS analysis. The left panel presents the absolute errors computed for chosen values of $k(0,0.2,0.4,0.6,0.8$, and 1) between the predicted and estimated stability scores with the PC analysis (dotted line, white circles) and the IS analysis (solid line, white
IS analysis

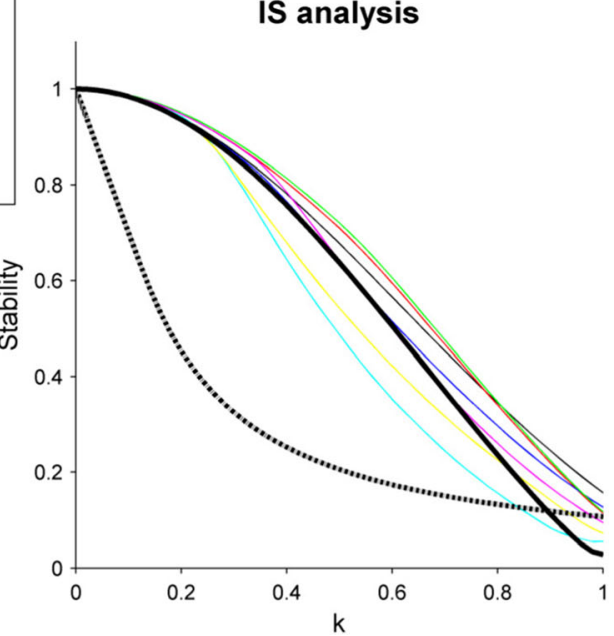

panel). The black dotted and solid lines represent the stability scores predicted by the $\mathrm{PC}$ analysis $\left(\mathrm{PC}_{\mathrm{pred}}\right)$ and the IS analysis ( $\mathrm{IS}_{\mathrm{pred}}$ ), respectively

In the following sections, we first attempt to identify the sources of the lack of accuracy and reliability of the PC analysis. Then we propose an extension of the IS analysis using optional inputs parameters that enable an even better estimate of stability. Finally we discuss how to improve the flexibility of the IS analysis and how to adjust it depending on the user's needs.

\section{A lack of accuracy and reliability of the PC analysis for estimating coordination stability}

Our simulation allows for comparing the extent to which the different calculations used by the PC analysis and the IS

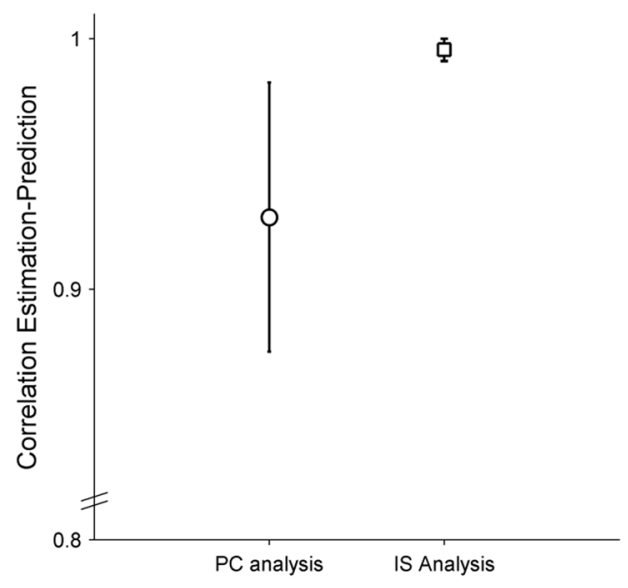

squares). The right panel shows the average computed correlation coefficients between the stability score functions predicted and estimated by the PC analysis (white circle) and by the IS analysis (white square) for each $m: n$ mode tested. Error bars represent the $95 \%$ confidence intervals 


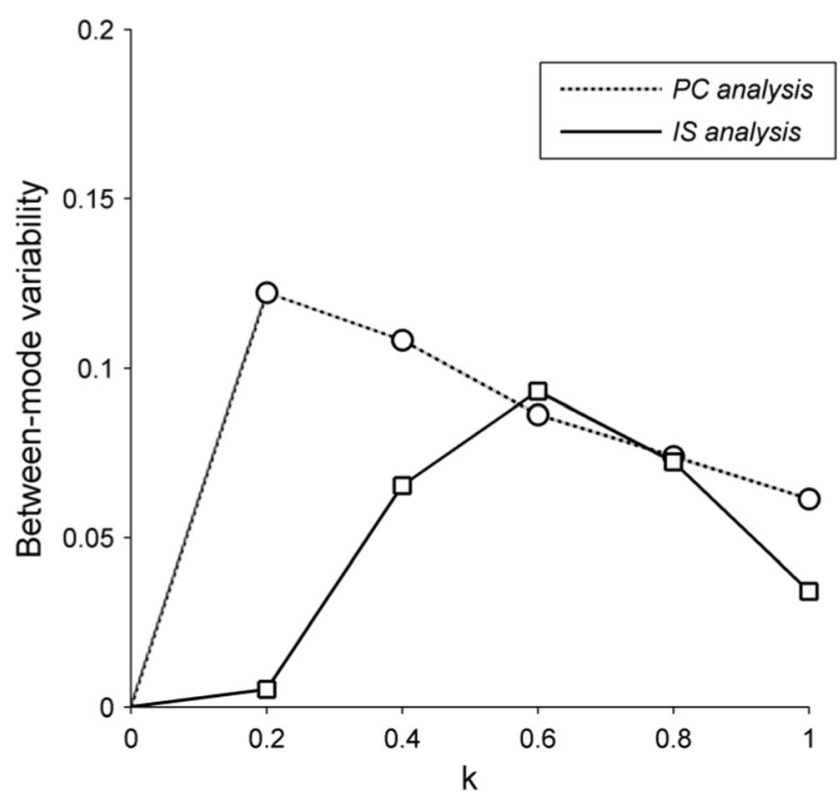

Fig. 6 Variability of the estimated stability scores across the seven $m: n$ modes tested as a function of $k$. The figure represents the standard deviations of the stability scores estimated at certain values of $k(0,0.2$, $0.4,0.6,0.8$, and 1 ) for the $m: n$ modes tested, by the PC analysis (dotted line, white circles) and the IS analysis (solid line, white squares)

analysis affect the estimate of coordination stability. In contrast to the IS analysis, the PC analysis first considers the computation of a standard relative phase time series that is independent of the mode of the coordination. That is, regardless of the mode of coordination produced, the relative phase time series is determined the same way (Eq. 8 in $\mathrm{S} 1$ Appendix). The mode of coordination is only used when estimating stability by assessing the fluctuations of the relative

Table 1 Percentages of correct identifications of the chosen $m: n$ mode by the PC analysis and the IS analysis

\begin{tabular}{|c|c|c|c|c|c|c|c|c|}
\hline \multirow[t]{2}{*}{ Chosen $m: n$ mode } & \multirow[t]{2}{*}{ Analysis } & \multicolumn{7}{|l|}{$k$} \\
\hline & & 0 & 0.04 & $\ldots$ & 0.88 & 0.92 & 0.96 & 1 \\
\hline \multirow[t]{2}{*}{$1: 1$} & $\mathrm{PC}$ & 100 & 100 & $\ldots$ & 100 & 100 & 100 & 100 \\
\hline & IS & 100 & 100 & $\ldots$ & 100 & 100 & 100 & 100 \\
\hline \multirow[t]{2}{*}{$1: 2$} & $\mathrm{PC}$ & 100 & 100 & $\ldots$ & 100 & 100 & 100 & 100 \\
\hline & IS & 100 & 100 & $\ldots$ & 100 & 100 & 100 & 84 \\
\hline \multirow[t]{2}{*}{$2: 1$} & $\mathrm{PC}$ & 100 & 100 & $\ldots$ & 100 & 100 & 100 & 100 \\
\hline & IS & 100 & 100 & $\ldots$ & 100 & 100 & 100 & 100 \\
\hline \multirow[t]{2}{*}{$1: 3$} & $\mathrm{PC}$ & 100 & 100 & $\ldots$ & 100 & 100 & 100 & 100 \\
\hline & IS & 100 & 100 & $\ldots$ & 100 & 100 & 100 & 76 \\
\hline \multirow[t]{2}{*}{$2: 3$} & $\mathrm{PC}$ & 100 & 100 & $\ldots$ & 100 & 100 & 100 & 100 \\
\hline & IS & 100 & 100 & $\ldots$ & 100 & 100 & 100 & 100 \\
\hline \multirow[t]{2}{*}{$3: 2$} & $\mathrm{PC}$ & 100 & 100 & $\ldots$ & 100 & 100 & 100 & 100 \\
\hline & IS & 100 & 100 & $\ldots$ & 100 & 94 & 86 & 80 \\
\hline \multirow[t]{2}{*}{$3: 1$} & $\mathrm{PC}$ & 100 & 100 & $\ldots$ & 100 & 100 & 100 & 100 \\
\hline & IS & 100 & 100 & $\ldots$ & 100 & 98 & 84 & 54 \\
\hline
\end{tabular}

phase time series. Here, the mode is essential, because it determines the lag at which the relative phase values are compared in the return map (see Eqs. 9 and 10; PC Step 3 in S1 Appendix). One possibility for the lack of accuracy and reliability of the PC analysis in comparison to the IS analysis is that the computation of the standard relative phase time series was affected by our manipulations of the time onsets of the component $\mathrm{A}$ in the simulation. Figure 7 shows the influence of the jitter used in the data simulation on computation of the relative phase time series, as calculated using the PC analysis and the IS analysis.

From Fig. 7, it is clear that if a jitter $J_{i}$ is applied at the time onset $t_{i}$ of component $\mathrm{A}$, the resultant relative phase computed via the $\mathrm{PC}$ analysis is influenced by the sign of the jitter. That is, the effects of the jitter are not symmetric: The relative phase computed for the jittered time onset $t_{i}$ differs depending on whether the jitter $J_{i}$ applied is positive or negative. Consider, for example, the jitter $\alpha$ applied on the time onset $t_{i}$ of component $\mathrm{A}$ in a 1:1 mode of coordination. It is clear that the resultant relative phase computed via the $\mathrm{PC}$ analysis is different if $\alpha$ is negative - that is, $R P\left(t_{i}-\alpha\right)=\frac{\alpha}{T+\alpha} * 2 \pi$ - or positive - that is, $R P\left(t_{i}+\alpha\right)=2 \pi$-where $T$ is the current cycle duration between $t_{i}$ and $t_{i+1}$. In contrast, the sign of $\alpha$ has no influence on the relative phase computed via the IS analysis: $R P\left(t_{i}-\alpha\right)=R P\left(t_{i}+\alpha\right)=\frac{\alpha}{T+\alpha} * 2 \pi$.

Another limitation shown in Fig. 7 is that for the PC analysis, the same jitter $J_{i}$ will have a different influence on the computation of the resultant relative phase, depending on the mode of the coordination. In comparison, the IS analysis provides a symmetric and consistent computation of the relative phase for any $m: n$ mode. To further understand the role of the computation of the relative phase on the lack of accuracy and reliability of the PC analysis in estimating coordination stability, we retested the PC analysis with the simulated data by modifying the computation of the standard relative phase time series in Eq. 8 (S1 Appendix), keeping only the fundamental requirement for the $\mathrm{PC}$ analysis to compute the relative phase independently of the coordination mode. To do so, we used the following expression instead of Eq. 8 (see S1 Appendix):

$R P=\theta_{B}-\theta_{A}$.

This corresponds to the calculation of the relative phase employed in the IS analysis when a 1:1 mode of coordination is considered (Eq. 13; S3 Appendix). The results are shown in Fig. 8. The estimation of the PC analysis clearly benefits from this change in relative phase computation, and it actually matches the prediction for the modes 1:1, 1:2, and 1:3. However, it noticeably overestimates the prediction for modes $m: n$ where $m \neq 1$. That is, the PC analysis still does not result in a correct estimate, even when we change the expression of relative phase to a more standard form. 


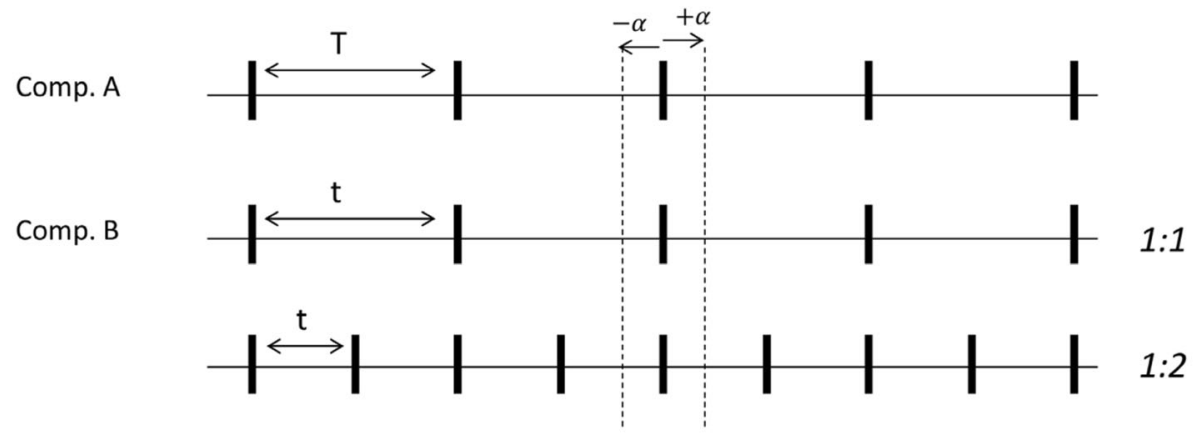

\begin{tabular}{|c|c|c|c|}
\hline \multirow{2}{*}{ Analysis } & \multirow{2}{*}{ Mode } & \multicolumn{2}{|c|}{ Jitter } \\
\hline & & $-\alpha$ & $+\alpha$ \\
\hline \multirow{2}{*}{ PC } & 1:1 & $R P=\frac{\alpha}{T+\alpha} * 2 \pi$ & $R P=\frac{t-\alpha}{T-\alpha} * 2 \pi=\frac{T-\alpha}{T-\alpha} * 2 \pi$ \\
\hline & $1: 2$ & $R P=\frac{\alpha}{T+\alpha} * 2 \pi$ & $R P=\frac{t-\alpha}{T-\alpha} * 2 \pi$ \\
\hline \multirow{2}{*}{ IS } & 1:1 & $R P=-\frac{\alpha}{t} * 2 \pi$ & $R P=\frac{\alpha}{t} * 2 \pi$ \\
\hline & $1: 2$ & $R P=-\frac{\alpha}{t} * 2 \pi$ & $R P=\frac{\alpha}{t} * 2 \pi$ \\
\hline
\end{tabular}

Fig. 7 Influences of variations in the time onset of component A on the relative phase calculations for the PC analysis and the IS analysis (see the text for details)

\section{Improving the estimate of stability in the IS analysis}

For the IS analysis, the calculated scores of stability did not completely match the score $I S_{\text {pred }}$ predicted by the method (the prediction of the dispersion of $\theta(k)$ using the mean resultant length technique; see Fig. 4, right panel). In the following paragraphs we discuss the influence of two factors with respect to their contributions to errors of estimation.

The first factor is related to the IS analysis itself, which considers multiple rational ratios $p: q$ for computing the relative phase time series (see IS Steps 2 and 3 in S3 Appendix). Each relative phase value at the time onsets $t_{i}$ and $t_{j}$ is computed in relation to the $p: q$ rational ratio that is locally identified. This definition of the relative phase is useful when no assumption is made about the mode of the coordination. In particular, it has the advantage of estimating an accurate phase coupling when two or more modes are performed within a dataset (Limitation 2 of the PC analysis in S2 Appendix). However, the analysis loses precision when only one mode is performed (as in the simulation above). To understand the role of this factor in the estimation of stability by the IS analysis, we recalculated it using only the most dominant rational ratio (e.g., $K R=1$ in Fig. 9). Figure 9 clearly shows an

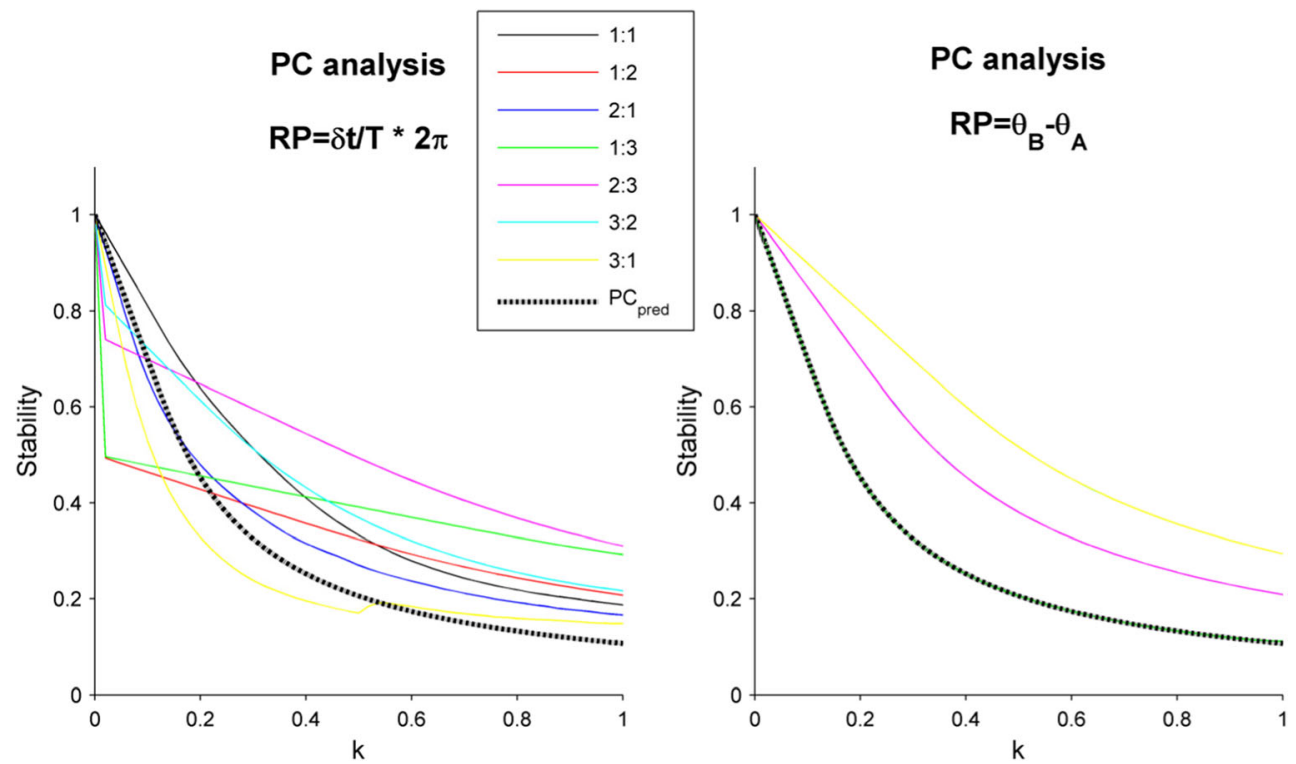

Fig. 8 Stability estimated with the PC analysis with $\mathrm{RP}\left(t_{j}\right)=\frac{\Delta t}{T} * 2 \pi$ (left panel; see Eq. 8 in S1 Appendix) or with $\mathrm{RP}\left(t_{j}\right)=\theta_{\mathrm{B}}\left(t_{j}\right)-\theta_{\mathrm{A}}\left(t_{j}\right)($ right panel; see Eq. 6 above). Note that the stability scores are given as a function of $k$ for each $m: n$ mode tested 

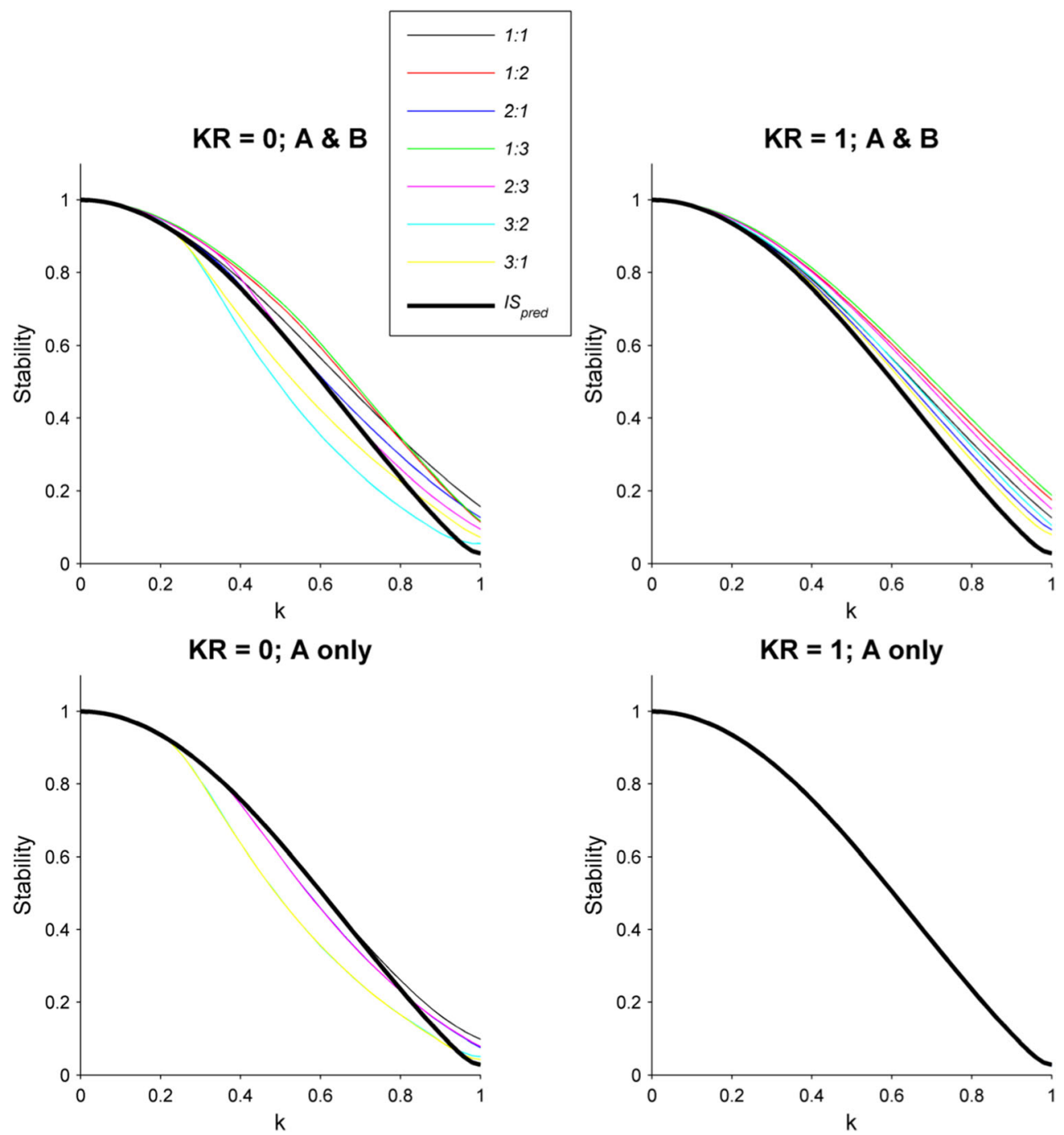

Fig. 9 Stability estimated with the IS analysis using the mean resultant length technique. Stability scores are given as a function of $k$ for each $m: n$ mode tested. Each graph plots the stability score IS $(k)$ estimated when processing the simulated time series using the IS analysis with different input parameters. $K R$ is the number of modes known a priori to be produced in the simulated data: 0 (No a priori knowledge; standard IS

improvement in the estimate of stability, since the estimated score is closer to the predicted score $I S_{\text {pred }}$ (top right panel, $K R=1$ ) than in the original analysis (top left panel, $K R=0$ ).

The second factor is related to our simulations, in which the variability of the time onsets $t_{i}$ was manipulated such that the relative phase computed for the time onsets $t_{i}$ was distributed over the $\left[-k^{*} \pi ; k^{*} \pi\right]$ interval. Note that a characteristic of the IS analysis is to systematically compute the relative phase time series for time onsets $t_{i}$ and $t_{j}$. A problem with this is that, in contrast to the relative phase values computed for the time onsets $t_{i}$ that were controlled to be distributed on the $\left[-k * \pi ; k^{*} \pi\right]$ interval, the relative phase values computed for the time onsets $t_{j}$ of component B were not controlled, and therefore were not necessarily distributed on such an interval. A second problem is that the number of time onsets $t_{j}$

analysis) or 1 (only one mode produced). "A \& B" indicates that the relative phase was considered for all time onsets $t_{i}$ and $t_{j}$ (standard IS analysis). "A only" means that the relative phase was considered for the time onsets $t_{i}$ only. The black lines represent the stability scores predicted by the IS analysis

changes as a function of the mode $m: n$ chosen for the simulation (see Fig. 2). That is, the number of $t_{j}$ onsets is greater for the mode $1: 3$ than for $1: 2,2: 3,1: 1,3: 2,2: 1$, and $3: 1$. Note that, accordingly, this ranking corresponds to the ranking of the modes by considering the maximal error between the estimated stability for $K R=1$ and the predicted $I S_{\text {pred }}$ (Fig. 9, top right panel). We tested the influence of the time onsets $t_{j}$ of oscillator B in the estimation of stability by testing the IS analysis while only considering the $t_{i}$ time onsets of the oscillator A ( $A$ only in Fig. 9). The results showed a perfect fit between the estimated stability and the predicted stability $I S_{\text {pred }}$ for all $m: n$ modes tested in the simulation (Fig. 9, bottom right panel).

Given the factors above, an optional input parameter $K R$ was included in the final version of the IS analysis for the case in which the user has a priori knowledge about the number of 
modes produced in the dataset (S5 Appendix). As we demonstrated above, knowing a priori the number of modes produced in the dataset enables the IS analysis to be more accurate. For $K R=0$, no assumption is made about the number of modes performed, and the mode and stability of the coordination are estimated as we explained in the standard description of the method (S3 Appendix). For $K R=x$, the analysis is applied by computing the relative phase time series according to the $x$ most dominant modes in the time series of rational ratios $R R_{n}$. For instance, if $x=2$, two modes are postulated to have been produced in the dataset, so for each $R R_{n}$ considered, the analysis computes a relative phase time series according to only the two most dominant modes. In other words, all real quotients $R Q\left(t_{i, j}\right)$ are assigned to one of these two most dominant modes. If $R Q\left(t_{i, j}\right)$ does not belong to a region of attraction of one of these two modes (for details, see PC Step 2 in S1 Appendix), it is automatically assigned to the closest one.

\section{The return map technique applied with the IS analysis}

The IS analysis uses the mean resultant length technique to assess the dispersion of the relative phase time series. This allows Limitation 3 of the PC analysis to be overcome (S2 Appendix). It should be noted, however, that this technique for estimating angular data dispersion is much less efficient if transitions occur in the mode in which the phases are coupled - for example, if the phases are locked in-phase and then switch to an antiphase phase-locking: The technique fails to identify such transitions, which results in an inexact
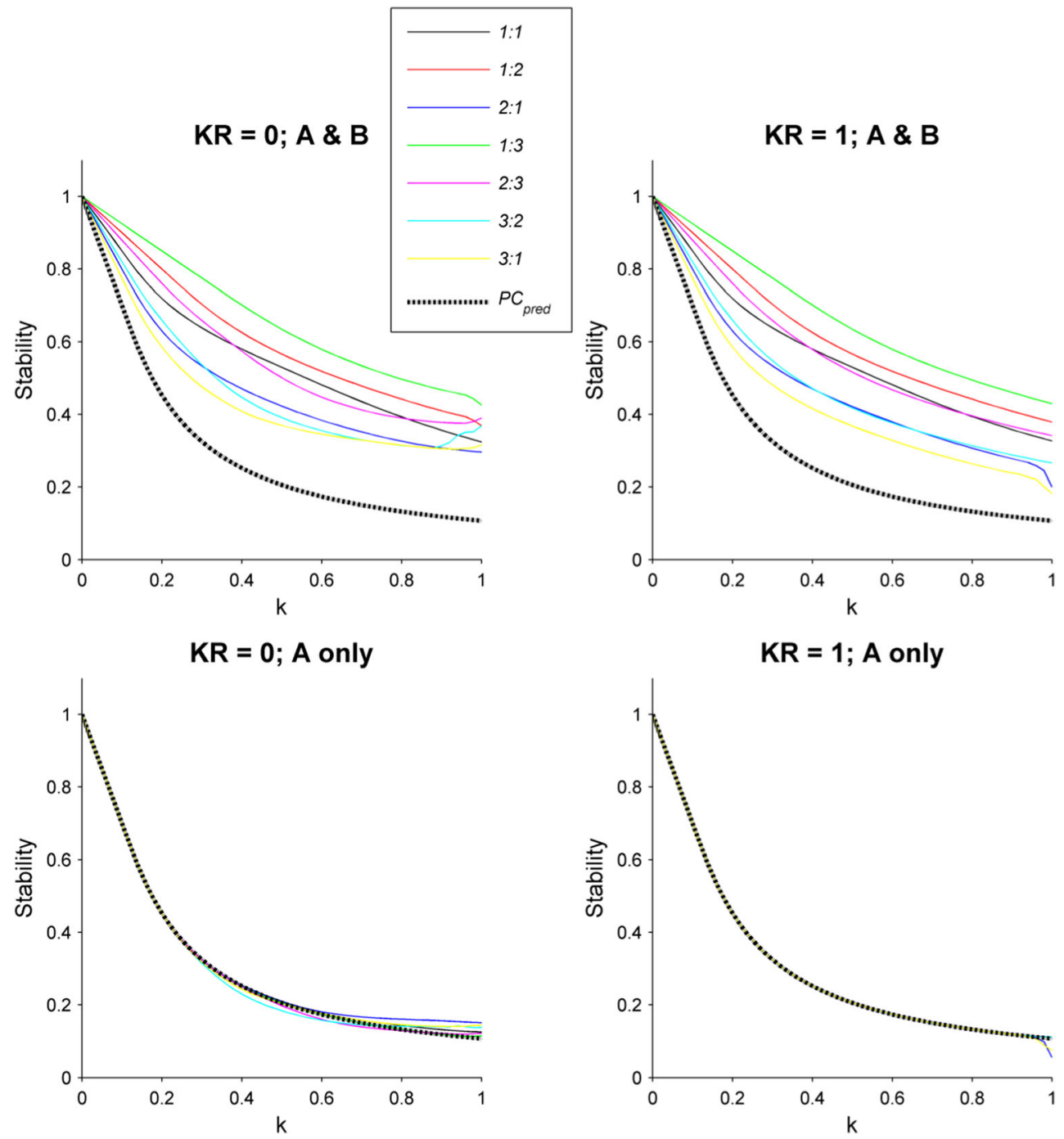

Fig. 10 Stability estimated with the IS analysis using the return map technique. Stability scores are given as a function of $k$ for each $m: n$ mode tested. Each graph plots the stability score estimated when processing the simulated time series using the IS analysis with the return map technique. $K R$ is the number of modes known a priori to be produced in the simulated data: 0 (No a priori knowledge; standard IS

analysis) or 1 (only one mode produced). "A \& B" indicates that the relative phase was considered for all time onsets $t_{i}$ and $t_{j}$ related to the components A and B (standard IS analysis). "A only" means that the relative phase was considered for the time onsets $t_{i}$ only. The black dotted lines represent the stability scores predicted with the return map technique 
estimation of stability. Interestingly, the return map technique used in the PC analysis for estimating the fluctuations of relative phase is sensitive to such a phenomenon. Therefore, an alternative for the IS analysis would be to use the return map technique to assess the fluctuations of the relative phase time series that have been computed in Step 2 of the IS analysis. Results from using such a procedure are shown in Fig. 10. Here, a perfect fit was found between the estimated stability scores and those predicted by the IS analysis using the return map technique for $K R=1$ and when only the time onsets of the component A were considered (see above for details). This perfect fit occurred no matter what $m: n$ mode was chosen for the simulation (Fig. 10, bottom right panel). Taking this into consideration, the implemented IS analysis provides the option of assessing the fluctuations of the relative phase time series with the return map technique.

\section{Flexibility in using the IS analysis}

An additional advantage of the IS analysis as compared to the PC analysis is that it is relatively simple to provide the user with different strategies for estimating the mode and stability, depending on his or her needs. The version described above considers stability as the variability of the phase coupling over the whole dataset. That is, the fluctuations of the phase coupling are examined without considering to which mode the phase coupling is related. Some researchers might disagree with this way of characterizing the dynamics of spontaneous coordinated behaviors, and might prefer, for instance, to examine the fluctuations of the phase coupling with respect to the mode performed. The IS analysis allows for such an estimate, because it is possible to select and estimate the fluctuations of the relative phase values related to one specific mode only. A global score can then be calculated by, for instance, weighting the stability scores computed for each mode as a function of the proportion of occurrences of the mode in the dataset.

\section{Conclusion}

The present work has introduced a new method, called the IS analysis, to estimate the mode and stability of spontaneous coordination. We showed that this method gives a more accurate and reliable estimate of stability than does the method currently most used in the area, the PC analysis. In addition, whereas the PC analysis is limited due to the assumptions it needs to make about the nature of the coordination, the IS analysis is not, because it does not require these conditions - that is, the IS analysis is balanced (in contrast to Limitation 1 of PC analysis), it is sensitive to changes in coordination modes (in contrast to Limitation 2 of PC analysis), and it is sensitive to phase drifts (in contrast to Limitation 3 of $\mathrm{PC}$ analysis). The IS analysis is also more flexible, because the user can adapt the final calculations depending on her or his needs and on the way that she or he likes to characterize the dynamics of spontaneous coordinated behaviors. Finally, the IS analysis can also be optimized with little a priori knowledge about the dataset - for example, if the number of modes performed is known (parameter $K R$ in the final version in $\mathrm{S} 5$ Appendix). The flexibility and accuracy of the IS analysis in estimating the stability of spontaneous coordinated behaviors open up new avenues for studying the weak coordinated behaviors produced in ecological situations.

\section{References}

Bak, P., Bohr, T., \& Jensen, M. H. (1985). Mode-locking and the transition to chaos in dissipative systems. Physica Scripta, 1985, 50-58.

Beek, P. J., Peper, C. E., \& van Wieringen, P. C. W. (1992). Frequency locking, frequency modulation, and bifurcations in dynamic movement systems. In Tutorials in motor behavior II (pp. 599-622). Amsterdam, The Netherlands: Elsevier.

Bramble, D. M., \& Carrier, D. R. (1983). Running and breathing in mammals. Science, 219, 251-256.

Cvitanovic, P., Shraiman, B., \& Söderberg, B. (1985). Scaling laws for mode lockings in circle maps. Physica Scripta, 32, 263-270.

Fisher, N. I. (1995). Statistical analysis of circular data. Cambridge, UK: Cambridge University Press.

Guevara, M. R., \& Glass, L. (1982). Phase locking, period doubling bifurcations and chaos in a mathematical model of a periodically driven oscillator: A theory for the entrainment of biological oscillators and the generation of cardiac dysrhythmias. Journal of Mathematical Biology, 14, 1-23.

Haken, H. (2013). Synergetics: Introduction and advanced topics. New York, NY: Springer Science \& Business Media.

Haken, H., Kelso, J. S., \& Bunz, H. (1985). A theoretical model of phase transitions in human hand movements. Biological Cybernetics, 51, 347-356.

Hamill, J., McDermott, W. J., \& Haddad, J. M. (2000). Issues in quantifying variability from a dynamical systems perspective. Journal of Applied Biomechanics, 16, 407-418.

Hardy, G. H., \& Wright, E. M. (1979). An introduction to the theory of numbers. Oxford, UK: Oxford University Press.

Hoffmann, C. P., Torregrosa, G., \& Bardy, B. G. (2012). Sound stabilizes locomotor-respiratory coupling and reduces energy cost. PLoS ONE, 7, e45206. doi:10.1371/journal.pone.0045206

Jammalamadaka, S. R., \& Sengupta, A. (2001). Topics in circular statistics. River Edge, NJ: World Scientific.

Jensen, M. H., Bak, P., \& Bohr, T. (1984). Transition to chaos by interaction of resonances in dissipative systems: I. Circle maps. Physical Review A, 30, 1960-1969.

Kelso, J. A. S. (1991). Multifrequency behavioral patterns and the phase attractive circle map. Biological Cybernetics, 64, 485-495.

Kelso, J. A. S. (1995). Dynamic patterns: The self-organization of brain and behavior. Cambridge, MA: MIT Press.

Kelso, J. A. S., \& de Guzman, G. C. (1988). Order in time: How the cooperation between the hands informs the design of the brain. In Neural and synergetic computers (pp. 180-196). Berlin, Germany: Springer.

Kelso, J. A. S., \& Jeka, J. J. (1992). Symmetry breaking dynamics of human multilimb coordination. Journal of Experimental Psychology: Human Perception and Performance, 18, 645-668. doi:10.1037/0096-1523.18.3.645 
Kelso, J. A. S., DeGuzman, G. C., \& Holroyd, T. (1991). Synergetic dynamics of biological coordination with special reference to phase attraction and intermittency. In H. P. Koepchen \& H. Haken (Eds.), Rhythms in physiological systems (pp. 195-213). Berlin, Germany: Springer.

Kelso, J. A. S., de Guzman, G. C., Reveley, C., \& Tognoli, E. (2009). Virtual partner interaction (VPI): Exploring novel behaviors via coordination dynamics. PLoS ONE, 4, e5749. doi:10.1371/journal. pone. 0005749

Kiefer, A. W., Riley, M. A., Shockley, K., Villard, S., \& Van Orden, G. C. (2009). Walking changes the dynamics of cognitive estimates of time intervals. Journal of Experimental Psychology: Human Perception and Performance, 35, 1532-1541.

Lagarde, J. (2013). Challenges for the understanding of the dynamics of social coordination. Frontiers in Neurorobotics, 7, 18. doi:10.3389/ fnbot.2013.0001

Lagarde, J., Zelic, G., \& Mottet, D. (2012). Segregated audio-tactile events destabilize the bimanual coordination of distinct rhythms. Experimental Brain Research, 219, 409-419. doi:10.1007/s00221012-3103-y

McDermott, W. J., Van Emmerik, R. E., \& Hamill, J. (2003). Running training and adaptive strategies of locomotor-respiratory coordination. European Journal of Applied Physiology, 89, 435-444.

Nourrit-Lucas, D., Zelic, G., Deschamps, T., Hilpron, M., \& Delignières, D. (2013). Persistent coordination patterns in a complex task after 10 years delay: subtitle: How validate the old saying "once you have learned how to ride a bicycle, you never forget!". Human Movement Science, 32, 1365-1378. doi:10.1016/j.humov.2013.07.005

O'Halloran, J., Hamill, J., McDermott, W. J., Remelius, J. G., \& Van Emmerik, R. E. (2012). Locomotor-respiratory coupling patterns and oxygen consumption during walking above and below preferred stride frequency. European Journal of Applied Physiology, 112, 929-940.

Peper, C. E., Beek, P. J., \& Van Wieringen, P. C. W. (1991). Bifurcations in polyrhythmic tapping: in search of Farey principles. In Tutorials in motor neuroscience (pp. 413-431). Amsterdam, The Netherlands: Springer.

Peper, C. L. E., Beek, P. J., \& van Wieringen, P. C. (1995). Frequencyinduced phase transitions in bimanual tapping. Biological Cybernetics, 73, 301-309.

Pikovsky, A., Rosenblum, M., \& Kurths, J. (2003). Synchronization: A universal concept in nonlinear sciences (Vol. 12). Cambridge, UK: Cambridge University Press.
Repp, B. H., \& Su, Y. H. (2013). Sensorimotor synchronization: A review of recent research (2006-2012). Psychonomic Bulletin \& Review, 20, 403-452. doi:10.3758/s13423-012-0371-2

Shockley, K., Baker, A. A., Richardson, M. J., \& Fowler, C. A. (2007), Articulatory constraints on interpersonal postural coordination. Journal of Experimental Psychology: Human Perception and Performance, 33, 201-208. doi:10.1037/0096-1523.33.1.201

Sternad, D., Turvey, M. T., \& Saltzman, E. L. (1999). Dynamics of 1:2 coordination: Generalizing relative phase to $n: m$ rhythms. Journal of Motor Behavior, 31, 207-223.

Tass, P., Rosenblum, M. G., Weule, J., Kurths, J., Pikovsky, A., Volkmann, J., ... Freund, H. J. (1998). Detection of $n: m$ phase locking from noisy data: application to magnetoencephalography. Physical Review Letters, 81, 3291-3294.

Treffner, P. J., \& Turvey, M. T. (1993). Resonance constraints on rhythmic movement. Journal of Experimental Psychology: Human Perception and Performance, 19, 1221-1237. doi:10.1037/00961523.19.6.1221

Turvey, M. T. (1990). Coordination. American Psychologist, 45, 938 953.

Van Emmerik, R. E., Rosenstein, M. T., McDermott, W. J., \& Hamill, J. (2004). A nonlinear dynamics approach to human movement. Journal of Applied Biomechanics, 20, 396-420.

Varlet, M., Marin, L., Lagarde, J., \& Bardy, B. G. (2011). Social postural coordination. Journal of Experimental Psychology: Human Perception and Performance, 37, 473-483.

Villard, S., Casties, J. F., \& Mottet, D. (2005). Dynamic stability of locomotor respiratory coupling during cycling in humans. Neuroscience Letters, 383, 333-338.

Zelic, G., Mottet, D., \& Lagarde, J. (2012). Behavioral impact of unisensory and multisensory audio-tactile events: Pros and cons for interlimb coordination in juggling. PLoS ONE, 7, e32308. doi: 10.1371/journal.pone.0032308

Zelic, G., Kim, J., \& Davis, C. (2015). Articulatory constraints on spontaneous entrainment between speech and manual gesture. Human Movement Science, 42, 232-245.

Zelic, G., Mottet, D., \& Lagarde, J. (2016). Perceptuo-motor compatibility governs multisensory integration in bimanual coordination dynamics. Experimental Brain Research, 234, 463-474. doi:10.1007/ s00221-015-4476-5

Zelic, G., Varlet, M., Kim, J., \& Davis, C. (2016). Influence of pacer continuity on continuous and discontinuous visuo-motor synchronisation. Acta Psychologica, 169, 61-70. 